\title{
Peritenon Extensor Tendon Inflammation in Psoriatic Arthritis Is an Enthesitis-related Lesion
}

\author{
Cristina Macía-Villa, Sandra Falcao, Marwin Gutierrez, Julio Medina, Hilde Berner Hammer, \\ and Eugenio De Miguel
}

\begin{abstract}
Objective. To analyze the association between enthesitis, synovitis, and peritenon extensor tendon inflammation (PTI) in psoriatic arthritis (PsA).

Methods. PsA patients with swelling of metacarpophalangeal joints were included. Greyscale and power Doppler (PD) were used for synovitis and PTI ultrasound identification. Madrid Sonographic Enthesis Index (MASEI) was used for enthesitis assessment. PD activity was evaluated using PD item of MASEI and PD Outcome Measures in Rheumatology (OMERACT) definition.

Results. Synovitis had no association with enthesitis. PTI was associated with PD MASEI and PD OMERACT. Only PD OMERACT showed a positive correlation with PTI.

Conclusion. In PsA, PTI is associated to enthesitis, as opposed to synovitis. (First Release June 1 2019; J Rheumatol 2019;46:1295-8; doi:10.3899/jrheum.180856)
\end{abstract}

Key Indexing Terms:

\section{PSORIATIC ARTHRITIS ULTRASOUND}

Enthesitis is a cornerstone of psoriatic arthritis (PsA) physiopathology. Synovitis is another landmark of PsA, considered secondary to enthesitis by some authors ${ }^{1}$. Metacarpophalangeal joint (MCPJ) swelling is a frequent finding in PsA, assumed to be caused by synovitis. However, peritenon extensor tendon inflammation (PTI) has emerged as another cause of MCPJ swelling, demonstrating to be a specific feature of PsA, of value in the differential diagnosis with other inflammatory diseases ${ }^{2,3}$. Some authors consider PTI an enthesitis-like lesion ${ }^{3,4,5,6}$ because of the relationship between the extensor tendon and the retinaculum pulley structure at $\mathrm{MCPJ}^{7}$, creating a functional enthesis with great similarities to the "enthesis organ" concept ${ }^{5}$.

Ultrasound (US) is a sensitive, specific, and reliable tool to evaluate structural and inflammatory changes in both

From the Department of Rheumatology, Hospital Universitario Severo Ochoa, Madrid; Department of Rheumatology, Hospital Clínico Universitario, Valladolid; Department of Rheumatology, Hospital Universitario La Paz, Madrid, Spain; Department of Rheumatology, Chronic Diseases Study Center (CEDOC), NOVA Medical School,

Universidade Nova de Lisboa (UNL), Lisbon; Centro Hospitalar de Lisboa Occidental, Lisbon, Portugal; Division of Musculoskeletal and Rheumatic Disorders, Instituto Nacional de Rehabilitación, Mexico City, Mexico; Department of Rheumatology, Diakonhjemmet Hospital, Oslo, Norway.

C. Macía-Villa, MD, Department of Rheumatology, Hospital Universitario Severo Ochoa; S. Falcao, MD, Department of Rheumatology, CEDOC, NOVA Medical School, UNL, and, Centro Hospitalar de Lisboa Ocidental; M. Gutierrez, MD, Division of Musculoskeletal and Rheumatic Disorders, Instituto Nacional de Rehabilitación; J. Medina, MD, Department of Rheumatology, Hospital Clínico Universitario; H. Berner Hammer, MD, PhD, Department of Rheumatology, Diakonhjemmet Hospital; E. De Miguel, MD, Department of Rheumatology, Hospital Universitario La Paz. Address correspondence to Dr. C. Macía-Villa, Department of

Rheumatology, Hospital Universitario Severo Ochoa, Av. de Orellana, s/n, 28911 Leganés, Madrid, Spain.E-mail: ccmacia@gmail.com

Accepted for publication January 17, 2019.

\section{TENDON SYNOVITIS ENTHESIS}

enthesis ${ }^{8}$ and synovium ${ }^{9}$, and we have previously shown its high reliability on evaluation of $\mathrm{PTI}^{10}$. To improve the pathophysiological understanding of PsA, US could be used to explore the role of PTI.

To our knowledge, the connection between PTI and enthesitis in PsA has not been studied. Our objective was to examine the association between both PTI and synovitis in MCPJ with enthesitis in patients with PsA, using the enthesis US score Madrid Sonographic Enthesis Index (MASEI) ${ }^{11}$ as well as power Doppler (PD) MASEI and PD Outcome Measures in Rheumatology (OMERACT) definitions.

\section{MATERIALS AND METHODS}

Study population. This is a posthoc study of a previous publication from our group ${ }^{10}$, which included consecutive nonselected patients with PsA fulfilling ClASsification for Psoriatic ARthritis (CASPAR) criteria ${ }^{12}$ with clinical swelling of at least one of the 2nd to 5th MCPJ. In addition to the MCPJ assessment, we performed an enthesis US examination using MASEI. Clinical examination was performed by a rheumatologist before US assessment. Patients $<18$ years and those with explanations other than PsA for MCPJ swelling were excluded. Demographic, clinical, and laboratory data were collected. The study was approved by the local ethics committee (Hospital Clínico Valladolid, PI 15-275). Informed consent was obtained from all patients according to the Declaration of Helsinki.

US settings. All US examinations were performed by an expert rheumatologist blinded to clinical data. A MyLab 70 XVG machine (Esaote S.p.A.) with a 13-MHz linear transducer was used. PD settings were as follows: pulse repetition frequency $750 \mathrm{~Hz}$, wall filter 3, persistence 4, and Doppler frequency 7.1 MHz. Color gain was set just below the level of noise. Videos lasting 3 to $5 \mathrm{~s}$ were recorded both for MCPJ and enthesis for posterior reliability assessments.

MCPJ US assessment. As previously reported ${ }^{10}$, the dorsal aspects of $2 \mathrm{nd}$ to 5 th MCPJ of both hands were examined by US with the patient seated in front of the sonographer, with hands lying in prone position on a table. Both longitudinal and transverse scans were performed, moving the transducer from proximal to distal, and from radial to ulnar sides of MCPJ dorsal surface. Plenty of gel was used to avoid compression. PTI was defined as a

Personal non-commercial use only. The Journal of Rheumatology Copyright @ 2019 . All rights reserved. 
hypoechoic image surrounding the extensor digitorum tendon with or without PD signal ${ }^{2,3,13}$, and synovitis based on the OMERACT definition ${ }^{14}$. At least 16 videos per patient were obtained, and in each joint, presence or absence of PTI and synovitis in greyscale (GS) and PD modes was scored. As previously described, reliability assessment was performed among 5 readers, and consensus of at least 3 was defined as the true result ${ }^{10}$.

Enthesis US assessment. The 6 entheses included in the MASEI index (bilateral triceps, quadriceps, proximal and distal patellar, Achilles tendons, and proximal insertion of plantar aponeurosis) and the elementary lesions included (structure, thickening, erosion, enthesophytes, PD, and bursa) were evaluated in longitudinal and transverse views, obtaining at least 24 videos per patient. In addition to the MASEI PD item (defined as PD signal in cortical bone profile, intratendon, or bursa on the enthesis insertion area), OMERACT definition for PD in enthesis (PD signal at enthesis $\leq 2 \mathrm{~mm}$ to the cortical bone profile insertion $)^{15}$ was also evaluated as present or absent. Reliability assessment was performed among 3 readers, and consensus of at least 2 was defined as the true result.

Statistical analysis. Quantitative variables are given as mean (SD). Intraclass correlation coefficient (ICC) with $95 \%$ CI was calculated for the reliability analysis of MASEI based on a mean rating $(\kappa=3)$, absolute agreement, 2-way mixed effect model. To take into account the observed low prevalence of the PD subtypes, a prevalence and bias-adjusted $\kappa$ (PABAK) was used to evaluate its reliability. PTI and synovitis reliability was calculated by Cohen's $\kappa$ test. Student $t$ test for independent samples was used to compare continuous variables and chi-square for qualitative variables. Correlations were calculated with Spearman $\varrho$ test. SPSS statistical package version 20 (SPSS Inc.) for statistical analysis and Stata version 12 (StataCorp.) for PABAK analysis were used.

\section{RESULTS}

Patient characteristics. Twenty-seven PsA patients were included. Clinical characteristics are summarized in Table 1. MCPJ US assessment. As previously described ${ }^{10}$, a total of 216 MCPJ were evaluated clinically and by US. PTI PD was found in 18 patients $(66.7 \%)$ and 38 joints (17.6\%), and PTI

Table 1. Clinical features of the patients.

\begin{tabular}{lc}
\hline Characteristics & Values \\
\hline Patients, $\mathrm{n}$ & 27 \\
Men & $17(63)$ \\
Women & $10(37)$ \\
Age, yrs & $56 \pm 11$ \\
Disease duration, mos & $109 \pm 101$ \\
Type of psoriasis & \\
First-degree relative & $1(3.7)$ \\
Skin psoriasis & $10(37.1)$ \\
Nail psoriasis & $1(3.7)$ \\
Skin and nail psoriasis & $15(55.5)$ \\
Type of PsA & \\
Peripheral & $21(78)$ \\
Axial and peripheral & $6(22)$ \\
CRP, mg/l* & $8.3 \pm 8.2$ \\
ESR, mm/h* & $21.9 \pm 19.3$ \\
DAS28-CRP* & $3.6 \pm 0.9$ \\
DAS28-ESR* & $3.9 \pm 1.2$ \\
\hline
\end{tabular}

Values are $\mathrm{n}(\%)$ or mean $\pm \mathrm{SD}$ unless otherwise specified. $* \mathrm{CRP}, \mathrm{ESR}$, and their respective DAS28 calculations were available in only 18 patients. PsA: psoriatic arthritis; CRP: C-reactive protein; ESR: erythrocyte sedimentation rate; DAS28: 28-joint count Disease Activity Score.
GS in 19 patients $(70.3 \%)$ and 41 joints $(18.9 \%)$. Synovitis PD was found in 18 patients (66.7\%) and 41 joints (18.9\%), and synovitis GS in 23 patients $(85.1 \%)$ and 63 joints $(29.16 \%)$. Both PTI and synovitis US achieved good reliability ${ }^{10}$.

Enthesis US assessment. A total of 324 entheses were scanned by US. Mean (SD) MASEI score was 30.62 (13.89). PD MASEI was found in 59/324 (18.2\%) entheses and PD OMERACT in 33/324 (10.2\%). The interreader reliability achieved for MASEI was excellent with ICC 0.918 (0.846-0.960). The interreader reliability of the PD subtypes was also excellent with PABAK $0.860(0.756-0.960)$ for PD MASEI and 0.864 (0.759-0.962) for PD OMERACT.

Association and correlation between PTI, synovitis, MASEI, and PD subtypes. Results are shown in Table 2 and Table 3. $\mathrm{PD}$ and GS synovitis were not associated with MASEI, any of its items, or with PD OMERACT. However, both PD and GS PTI demonstrated significant association with PD MASEI and PD OMERACT. PTI GS also showed significant association with erosions. PD OMERACT showed a positive correlation with PTI PD and GS. PTI, both PD and GS, showed a significant association with synovitis PD (p 0.009 and p 0.037 , respectively). GS synovitis had no association with PD or GS PTI (p 0.055 and p 0.334, respectively).

\section{DISCUSSION}

According to the synovioentheseal mode ${ }^{16}$, enthesitis is the initial site of inflammation in PsA, positioning synovitis as a secondary lesion. In a previous study we demonstrated that MCPJ swelling in PsA has 2 etiopathological lesions: synovitis and $\mathrm{PTI}^{10}$. In the present study we tried to clarify a previously unevaluated topic: whether PTI is an enthesitis-related lesion. We found that synovitis at MCPJ was not associated with enthesitis. However, PTI showed a significant association with active enthesitis (PD MASEI, PD OMERACT) and erosions. The reason for evaluating PD subtypes is because we still do not have absolute agreement about which zone to restrict US enthesis lesions meaning inflammation ${ }^{15}$, but there is full agreement about PD as the main indicator of active enthesitis.

The extensor tendon at MCPJ level with its stabilizing wraparound band system and the local sesamoid fibrocartilage is considered a functional enthesis by some authors $3,4,5,6$. Our findings are in agreement with this hypothesis and with the concept of an entheseal organ ${ }^{5}$, representing pathologic changes that extend from the enthesis to the adjacent soft tissues. Moreover, although our study does not explore causality, our finding of the relationship between PTI and synovitis and between PTI and enthesitis, but not between enthesitis and synovitis, could be in agreement with the theory that the origin and epicenter of inflammation in spondyloarthritis is in the enthesis, considering synovitis as a secondary feature to enthesitis ${ }^{1,17}$.

To our knowledge, there is no evidence of association

Personal non-commercial use only. The Journal of Rheumatology Copyright $\odot$ (2019. All rights reserved 
Table 2. Association between PTI, synovitis, MASEI, and PD subtype.

\begin{tabular}{|c|c|c|c|c|c|c|c|c|c|c|c|c|}
\hline & \multicolumn{3}{|c|}{ PTI PD } & \multicolumn{3}{|c|}{ Synovitis PD } & \multicolumn{3}{|c|}{ PTI Greyscale } & \multicolumn{3}{|c|}{ Synovitis Greyscale } \\
\hline & Present & Absent & $\mathrm{p}$ & Present & Absent & $\mathrm{p}$ & Present & Absent & $\mathrm{p}$ & Present & Absent & $\mathrm{p}$ \\
\hline MASEI & $32.44 \pm 15.62$ & $27 \pm 9.33$ & 0.270 & $32 \pm 16.16$ & $27.89 \pm 7.72$ & 0.380 & $33.15 \pm 15.49$ & $24.6 \pm 6.43$ & 0.054 & $30.78 \pm 14.54$ & $29.75 \pm 11.02$ & 0.876 \\
\hline Thickness MASEI & $5.5 \pm 3.34$ & $5.66 \pm 3.42$ & 0.906 & $5.88 \pm 3.12$ & $4.88 \pm 3.75$ & 0.503 & $5.84 \pm 3.57$ & $4.87 \pm 2.64$ & 0.447 & $5.34 \pm 3.25$ & $6.75 \pm 3.86$ & 0.533 \\
\hline Erosion MASEI & $2.83 \pm 5.19$ & $0.66 \pm 2$ & 0.133 & $2.66 \pm 5.23$ & $1 \pm 2.12$ & 0.253 & $3 \pm 5.09$ & $0 \pm 0$ & 0.019 & $2.08 \pm 4.73$ & $2.25 \pm 2.87$ & 0.928 \\
\hline Calcification MASEI & I $6.44 \pm 2.57$ & $7.33 \pm 3.12$ & 0.473 & $6.77 \pm 2.81$ & $6.66 \pm 2.73$ & 0.923 & $6.68 \pm 2.70$ & $6.87 \pm 2.99$ & 0.879 & $6.69 \pm 2.8$ & $7 \pm 2.7$ & 0.846 \\
\hline PD OMERACT & $1.61 \pm 1.33$ & $0.56 \pm 1.01$ & 0.033 & $1.39 \pm 1.46$ & $1 \pm 1$ & 0.426 & $1.57 \pm 1.30$ & $0.5 \pm 1.06$ & 0.040 & $1.26 \pm 1.35$ & $1.25 \pm 1.25$ & 0.988 \\
\hline
\end{tabular}

Results expressed as mean \pm SD. P values in bold face $(<0.05)$ considered statistically significant. PTI: peritenon extensor tendon inflammation; MASEI: Madrid Sonographic Enthesis Index; PD: power Doppler; OMERACT: Outcome Measures in Rheumatology.

Table 3. Correlation between PTI, synovitis, and MASEI items.

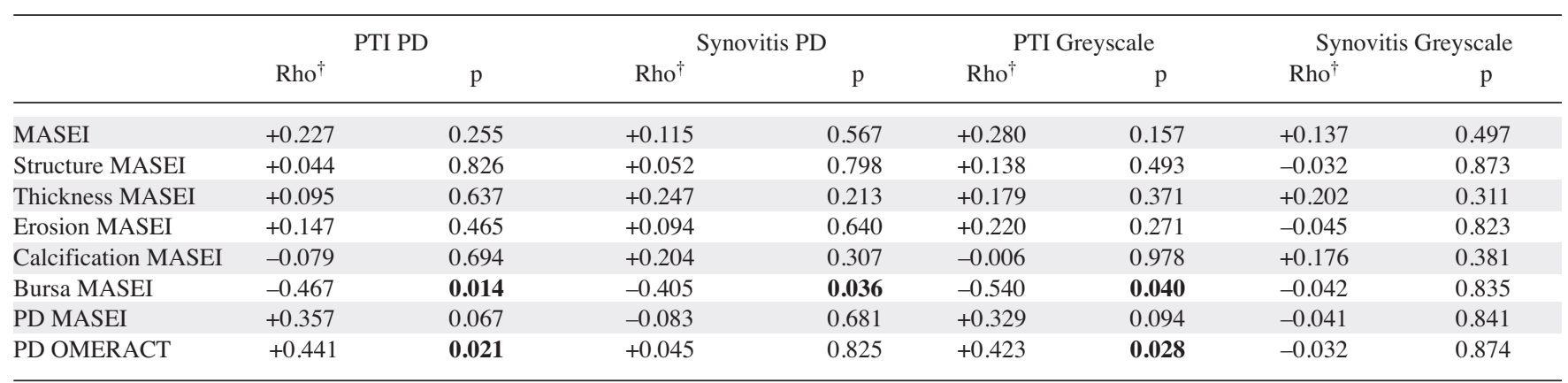

$\dagger$ Spearman rho value. P values in bold face $(<0.05)$ considered statistically significant. PTI: peritenon extensor tendon inflammation; MASEI: Madrid Sonographic Enthesis Index; PD: power Doppler; OMERACT: Outcome Measures in Rheumatology.

between MASEI and PTI. This is also the result of our study. A possible explanation is the different chronic and active lesions that make up this index, while PTI is an inflammatory lesion. On the contrary, when analyzing the association and correlation of PTI with PD, with PD being the most characteristic US lesion of active enthesitis ${ }^{18,19}$, we found statistical association between them. Our results support the hypothesis of PTI being an enthesitis-related lesion, mainly with its inflammatory changes.

Our study has some limitations. Sample size is low. Clinical enthesis data were not available. The reliability exercise was based on videos, not real scanning. However, a high-level machine was used, and the produced videos gave a close real-time examination. In addition, the reliability exercise included only sonographers with the highest level of competence (European League Against Rheumatism competency assessment level 2) and vast experience in evaluation and investigation in US enthesis lesions.

Our present study finds PTI to be associated with active enthesitis as opposed to MCPJ synovitis, which may support a functional association between PTI and enthesitis, and reinforces the role of PTI in PsA as an enthesis-related lesion. This possible association should be analyzed further in larger studies, as well as the implication of PTI on PsA treatment.

\section{REFERENCES}

1. McGonagle DG, Helliwell P, Veale D. Enthesitis in psoriatic disease. Dermatology 2012;225:100-9.

2. Gutierrez M, Filippucci E, Salaffi F, Di Geso L, Grassi W. Differential diagnosis between rheumatoid arthritis and psoriatic arthritis: the value of ultrasound findings at metacarpophalangeal joints level. Ann Rheum Dis 2011;70:1111-4.

3. Zabotti A, Salvin S, Quartuccio L, De Vita S. Differentiation between early rheumatoid and early psoriatic arthritis by the ultrasonographic study of the synovio-entheseal complex of the small joints of the hands. Clin Exp Rheumatol 2016;34:459-65.

4. Tan AL, Fukuba E, Halliday NA, Tanner SF, Emery P, McGonagle D. High-resolution MRI assessment of dactylitis in psoriatic arthritis shows flexor tendon pulley and sheath-related enthesitis. Ann Rheum Dis 2015;74:185-9.

5. Benjamin M, McGonagle D. The anatomical basis for disease localisation in seronegative spondyloarthropathy at entheses and related sites. J Anat 2001;199:503-26.

6. Filippou G, Di Sabatino V, Adinolfi A, Bertoldi I, Picerno V, Biasi $\mathrm{G}$, et al. No enthesis should be overlooked when psoriatic arthritis is suspected: enthesitis of the extensor digitorum tendons. J Rheumatol 2013;40:335.

7. Milz S, Putz R, Ralphs JR, Benjamin M. Fibrocartilage in the extensor tendons of the human metacarpophalangeal joints. Anat Rec 1999;256:139-45.

8. de Miguel E, Muñoz-Fernández S, Castillo C, Cobo-Ibáñez T, Martín-Mola E. Diagnostic accuracy of enthesis ultrasound in the diagnosis of early spondyloarthritis. Ann Rheum Dis 2011; 70:434-9. 
9. Grassi W, Salaffi F, Filippucci E. Ultrasound in rheumatology. Best Pract Res Clin Rheumatol 2005;19:467-85.

10. Macía-Villa C, Falcao S, Gutierrez M, Medina J, Hammer HB, De Miguel E. What is metacarpophalangeal joint swelling in psoriatic arthritis? Ultrasound findings and reliability assessment. Clin Exp Rheumatol 2018;36:896-9.

11. de Miguel E, Cobo T, Muñoz-Fernández S, Naredo E, Usón J, Acebes JC, et al. Validity of enthesis ultrasound assessment in spondyloarthropathy. Ann Rheum Dis 2009;68:169-74.

12. Taylor W, Gladman D, Helliwell P, Marchesoni A, Mease P, Mielants H; CASPAR Study Group. Classification criteria for psoriatic arthritis: development of new criteria from a large international study. Arthritis Rheum 2006;54:2665-73.

13. Husic R, Gretler J, Felber A, Graninger WB, Duftner C, Hermann J, et al. Disparity between ultrasound and clinical findings in psoriatic arthritis. Ann Rheum Dis 2014;73:1529-36.

14. Wakefield RJ, Balint PV, Szkudlarek M, Filippucci E, Backhaus M, D'Agostino MA, et al; OMERACT 7 Special Interest Group. Musculoskeletal ultrasound including definitions for ultrasonographic pathology. J Rheumatol 2005;32:2485-7.

15. Terslev L, Naredo E, Iagnocco A, Balint PV, Wakefield RJ, Aegerter
$\mathrm{P}$, et al; Outcome Measures in Rheumatology Ultrasound Task Force. Defining enthesitis in spondyloarthritis by ultrasound: results of a Delphi process and of a reliability reading exercise. Arthritis Care Res 2014;66:741-8.

16. McGonagle D, Lories RJ, Tan AL, Benjamin M. The concept of a "synovio-entheseal complex" and its implications for understanding joint inflammation and damage in psoriatic arthritis and beyond. Arthritis Rheum 2007;56:2482-91.

17. Marzo-Ortega H, Tanner SF, Rhodes LA, Tan AL, Conaghan PG, Hensor EM, et al. Magnetic resonance imaging in the assessment of metacarpophalangeal joint disease in early psoriatic and rheumatoid arthritis. Scand J Rheumatol 2009;38:79-83.

18. D'Agostino MA, Aegerter P, Bechara K, Salliot C, Judet O, Chimenti MS, et al. How to diagnose spondyloarthritis early? Accuracy of peripheral enthesitis detection by power Doppler ultrasonography. Ann Rheum Dis 2011;70:1433-40.

19. Zabotti A, Bandinelli F, Batticciotto A, Scirè CA, Iagnocco A, Sakellariou G, et al; Musculoskeletal Ultrasound Study Group of the Italian Society of Rheumatology. Musculoskeletal ultrasonography for psoriatic arthritis and psoriasis patients: a systematic literature review. Rheumatology 2017;56:1518-32. 